\title{
Poorly differentiated thyroid carcinoma: an incubating entity
}

\author{
Peter M. Sadow ${ }^{1,2 *}$ and William C. Faquin ${ }^{1,2}$ \\ 1 Pathology Service, Massachusetts General Hospital, Boston, MA, USA \\ 2 Department of Pathology, Harvard Medical School, Boston, MA, USA \\ *Correspondence: psadow@partners.org
}

Estimates for 2012 reveal thyroid cancer as the fifth most expected malignancy in American women. Although thyroid cancer is not infrequently diagnosed, it rarely bests its host. We understand a great deal about well-differentiated thyroid cancers, including carcinomas of the thyrocyte and parafollicular c-cells. We have identified a number of mutations and gene rearrangements responsible for familial and sporadic tumors. We have postulated about mechanisms of spread and are able to predict biological behaviors of particular cancer types. However, gray zones remain, and as physicians and scientists, we are never comfortable with these gray zones, as they potentially contain magical epitopes for disease eradication and serve as pesky reminders that medicine is not an exact science. In thyroid cancer, poorly differentiated thyroid carcinomas (PDTC) represent the bridge between more well differentiated malignancies of thyrocytes and the undifferentiated (anaplastic) thyroid carcinomas. Six years ago in Turin, a group of expert surgical pathologists set the stage for refining the diagnosis of PDTC, as introduced by the WHO in 2004, rather than it serving as a diagnosis for those entities which had "high grade features" but did not fit neatly into one of the other defined categories. The conundrum that now exists is the incubation period between the birth of this now well-defined category of thyroid malignancy and the application of the diagnostic criteria, outcomes going forward, and how this diagnosis becomes translated into action by clinicians treating the entity.

\section{WORLD HEALTH ORGANIZATION CLASSIFICATION OF ENDOCRINE TUMORS, 2004}

The WHO blue books are the gold standard for diagnostics in surgical pathology. Although the literature churns out an abundance of new data and potential classification schemes, these publications of the WHO are the cornerstones of ana- tomic pathology-driven diagnostics until they are subsequently updated by designated subspecialist pathologists of the next edition. The most recent classification of endocrine tumors was published in 2004, in which poorly differentiated thyroid carcinomas are introduced as a diagnostic entity (Sobrinho-Simões et al., 2004). PDTC are defined as "follicular neoplasms that show limited evidence of structural follicular cell differentiation and occupy both morphologically and behaviorally an intermediate position between differentiated (follicular and papillary carcinomas) and undifferentiated (anaplastic) carcinomas." This classification has been intended to combine various definitions of PDTC that have been circulating in the literature and diagnostic surgical pathology reports for many years with many overlapping features but varying degrees of broad interpretation (Sakamoto et al., 1983; Carcangiu et al., 1984; Akslen and LiVolsi, 2000; Sobrinho-Simões et al., 2002; Hiltzik et al., 2006).

\section{TURIN, 2006}

About 6 years ago, a group of expert thyroid surgical pathologists gathered in Turin, Italy, to finally hash out what would be considered by consensus to be reproducible criteria for the diagnosis of poorly differentiated thyroid carcinoma (Volante et al., 2007). The analyzed cohort consisted of 83 cases assembled from Europe (Italy), Japan, and the United States with a representative panel consisting of 12 thyroid pathologists. After a lively consensus, the published paper reported a diagnostic scheme, represented by the unshaded areas in Figure 1 (Volante et al., 2007). As part of the diagnostic algorithm, the tumor would first need to be deemed malignant. The growth patterns were those previously associated with PDTC, including solid, trabecular, or insular (STI) growth. The insular growth pattern is most familiar to pathologists and non-pathologists, with insular carcinoma often thought of as a high grade carcinoma with extensive necrosis, increased mitoses, broad infiltration of the surrounding thyroid tissue and beyond with a poor prognosis (Carcangiu et al., 1984). Solid and trabecular growth are also quite familiar to surgical pathologists, and these two patterns have diverse context. Most solid-patterned thyroid lesions represent follicular adenomas or benign, unencapsulated adenomatous nodules, but they may also represent solid-type papillary carcinoma, follicular carcinoma, or PDTC. Trabecular growth is largely seen in adenomas, and there is a well-known malignant mimic, the hyalinizing trabecular tumor, which is usually small $(<1 \mathrm{~cm})$, with a fingerprint-like appearance that may contain frequent intranuclear pseudoinclusions with low biological potential (Thompson, 2011). However, less frequently, trabecular growth, especially in large, cellular lesions, is ominous appearing, and may raise the possibility of a more biologically aggressive lesion.

The Turin criteria for the diagnosis of PDTC are an easy to follow flow chart (Figure 1, unshaded), which arrive at a diagnosis following a series of exclusions. The presence of STI growth is essential, and without this pattern, the tumor is immediately excluded from representing PDTC. If STI growth is present, but the tumor shows largely typical nuclear features of papillary thyroid carcinoma (PTC), the tumor is excluded. If no features of PTC are identified, the tumor is assessed for the presence of any of each of mitoses ( $\geq 3$ mitoses per $10 \mathrm{hpf}$ ), convoluted nuclei, or necrosis. Any or all of these features is then sufficient for a diagnosis of PDTC. If not, the lesion is deemed to be a well-differentiated follicular thyroid carcinoma.

These criteria appear simple. However, the first criterion present on the flow chart is that the lesion is a "malignant thyroid tumor of follicular cells." Of note, encapsulation is not a part of the diagnostic scheme. 


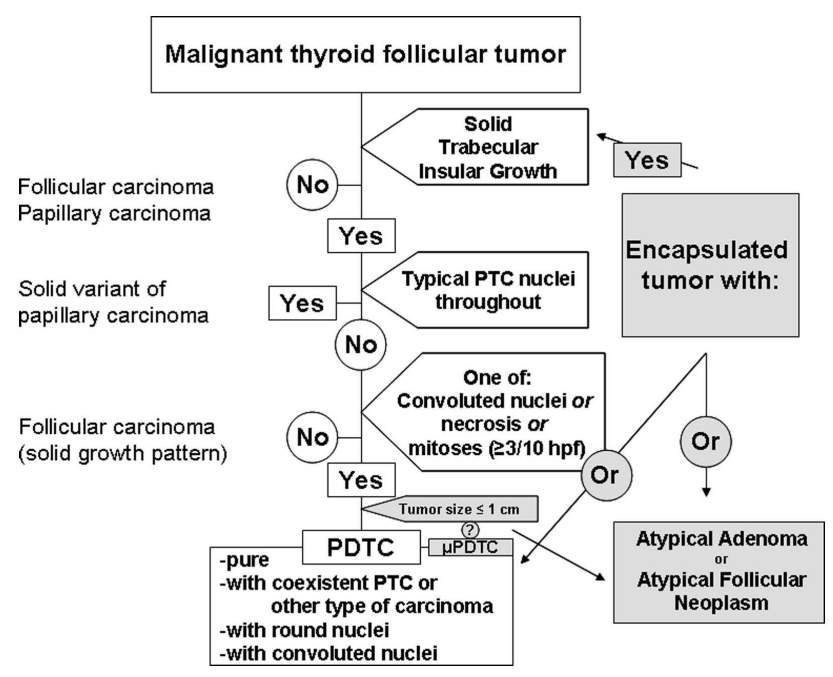

FIGURE 1 | Diagnosis of poorly differentiated thyroid carcinoma based upon the Turin proposal (unshaded). Lesions on the periphery pending inclusion criteria (shaded).

So, even with these clear definitions of PDTC, there exists an additional gray zone, that of the encapsulated follicular tumor with increased mitoses or focal necrosis (Figure 1, shaded). Convoluted nuclei are well-defined by the Turin consensus, but convoluted nuclei alone, without the aid of increased mitoses or necrosis, might be viewed as a subjective challenge to nonthyroid pathology experts. Additionally, how should pathologists interpret solitary lesions that meet the Turin criteria but are subcentimeter?

\section{ENCAPSULATED LESIONS}

As mentioned, some encapsulated lesions involve a diagnostic gray zone, as benign lesions, due to size, duration, or external stimuli, may contain features of PDTC. However, depending upon the subjective interpretation of the tumor's malignancy, there is room for broad interpretation. Is the tumor malignant because there is necrosis and mitoses? Or, is the tumor malignant, and therefore the presence of mitoses and/or necrosis appoints that lesion to be a PDTC? We know of encapsulated thyroid carcinomas, specifically, follicular variant of papillary carcinoma, that do not require capsular invasion to qualify as malignant. Biological potential aside, is an encapsulated lesion with convoluted nuclei, necrosis, or increased mitoses a PDTC or an atypical neoplasm of uncertain biological potential
(Figure 1, shaded; Rivera et al., 2010a)? This is unclear and untested, waiting to be fleshed out by retrospective, prospective, and collective data.

\section{STATE OF THE UNION}

Incidence of thyroid carcinomas is increasing, with 2012 expected to have thyroid carcinoma as the fifth leading cause of cancer in women (Siegel and Jemal, 2012). Improved classification will require more detailed information for treatment and outcome, with PDTC portending an intermediate prognosis between well-differentiated and undifferentiated thyroid neoplasms (Siironen et al., 2010). Since the Turin proposal, studies have emerged using these new criteria. In a single molecular study, 65 cases selected by Turin criteria were analyzed for mutations in $B R A F, N, K$, and HRAS, as well as translocation of RET/ PTC1, 3, and PAX8/PPAR $\gamma$. The majority of mutations (15/65) were found in RAS (largely NRAS), with a single mutation found in $B R A F$ (V600E). The single BRAF mutation was found in a PDTC associated with tall cell PTC (Volante et al., 2009). No other mutations were identified. This same paper looked at p53 by immunohistochemistry, with expression in $~ 30 \%$ of tumors, but accounting for less than 20\% of tumor staining, making mutated TP53 unlikely to be a major contributor. TP53 is well-known to be mutated in undifferentiated (anaplastic) carcinomas (Lavra et al.,
2009) and though reported or discussed in other cohorts of PDTC (Malaguarnera et al., 2007; Nambiar et al., 2011), it has not been well-studied in a significant postTurin cohort. Some estimates have placed mutations here at about 20-30\% (Nikiforov and Nikiforova, 2011), but most of the large TP53 mutational studies were performed in the early to mid-1990s (Donghi et al., 1993; Fagin et al., 1993; Dobashi et al., 1994). In an additional large study with collaborating authors from the aforementioned group, this cohort validated the Turin criteria in a retrospective fashion using cases from the United States and Italy, showing the addition of one molecular/immunohistochemical marker, IMP3, to be an indicator of poor prognosis in PDTC (Asioli et al., 2010). The study also reiterated NRAS to be most commonly mutated in PDTC, including the prior study (about $20 \%$ of cases).

\section{SPECIFICITY OF DIAGNOSIS IN PDTC}

Ultimately, the recent reclassification of PDTC is intended to improve patient care, with more uniform, accurate reporting. Importantly, the criteria exist to link objective findings with clinical outcomes, biological potential, and hopefully, following additional study, molecular mechanisms with targeted treatment modalities and improved outcomes. For instance, oncocytic neoplasms were excluded in the Turin proposal. However, in a recent validation by the Mayo group in collaboration with the Turin group, about $1 / 3$ of tumors had oncocytic features (Asioli et al., 2010). Also, a very recent study reviewing a large cohort of aggressive thyroid carcinomas (129 with known recurrence or death) identified 18 oncocytic PDTC (Dettmer et al., 2012). However, this group does not distinguish the 18 oncocytic PDTC from other oncocytic (Hürthle cell) carcinomas present among the 129 cases, and the majority $(16 / 18)$ of these cases were included due to necrosis, in addition to STI growth pattern. What constitutes convoluted nuclei in this group (18/18), with oncocytic nuclei generally thought of as round with prominent, centrally located nucleoli, is unclear.

\section{MISSING LINK}

Well-differentiated thyroid carcinomas of follicular origin, including PTCs and follicular thyroid carcinomas, have several known mutations or translocation events. 
PTCs have mutations in BRAF, occasional $R A S$ subtypes, and may have translocations of RET/PTC 1,2 (Nikiforov and Nikiforova, 2011). Follicular carcinomas can have aberrations of RAS or PAX8/PPAR $\gamma$ (Nikiforov and Nikiforova, 2011). Additional exceptions exist, but these are certainly most common for those who work with patients with thyroid cancer. Undifferentiated (anaplastic) thyroid carcinomas (ATC), in addition to possible prior BRAF or RAS mutations, may harbor additional mutations in TP53 or beta-catenin (CTNNB1; Nikiforov and Nikiforova, 2011). This mechanism of transformation is thought to be a multihit hypothesis, as ATC with associated more well-differentiated components have been shown to have mutations in BRAF in the well-differentiated component, with the additional CTNNB1 or TP53 mutation seen only in the undifferentiated areas (Nikiforov, 2004; Quiros et al., 2005).

However, this is not how we generally encounter these cancers. Most, seen microscopically, do not have a nice transition for us to identify tumor origin. Additionally, we may discover late recurrences of welldifferentiated tumors as something more sinister, often after some treatment modality other than surgical excision.

\section{WELL-DIFFERENTIATED CARCINOMAS WITH HIGH GRADE FEATURES}

We would like to briefly discuss the concept of a well-differentiated carcinoma with high grade features. Although papillary-type PDTC can show concurrent cancer types, many cancers exist as majority PTCs with foci of high grade changes, such as necrosis, focal loss of papillary-type nuclei, and foci with overall aberrant morphology (Rivera et al., 2010a,b). Widely invasive follicular or Hürthle cell carcinoma may have associated foci showing necrosis or increased mitoses, and by their nature, they are often associated with the STI growth pattern. Likely, this is why so many of the subsequently studied tumors post-Turin have RAS mutations (Ricarte-Filho et al., 2009; Volante et al., 2009; Asioli et al., 2010). This tumor is not entirely poorly differentiated, as you can see a more well-differentiated component to it, but drawing a definitive diagnostic line between FTC and PDTC remains challenging. The truth is that we call these tumors many things and often diagnose them descriptively, presenting challenges for our clinical colleagues, who would like to rely more and more often on molecular data for individualized care and potential targeted therapies, some of which have been approached with animal models (Nucera et al., 2010; Chakravarty et al., 2011), whereas some authors propose we scrap our current classification system entirely (Kakudo et al., 2012). Is this descriptive diagnosis preventing an appropriate accounting of disease incidence, or are we displaying the limits of our current knowledge base while additional molecular and proteomic data are accrued? We would argue for the latter.

\section{VALIDATION}

As the Turin criteria were released 5 years ago (Volante et al., 2007), there have been a few published validation studies (Volante et al., 2009; Asioli et al., 2010), including a recent study of oncocytic PDTC (Dettmer et al., 2012), controversial in that oncocytic tumors were excluded from the Turin study set. The majority of the discussion of the new criteria for PDTC is from a respectable cadre of experts (Bongiovanni et al., 2009; Garcia-Rostan and SobrinhoSimões, 2010; Volante and Papotti, 2010; Dettmer et al., 2011; Nambiar et al., 2011; Tallini, 2011).

\section{CONCLUSION}

The past decade has seen a shift in diagnosis of follicular-patterned thyroid lesions, including high grade lesions. The publication in 2004 of the WHO blue book on endocrine tumors introduced poorly differentiated thyroid carcinoma as a distinct diagnostic entity. This was further welldelineated in Turin in 2006, providing tangible criteria for the everyday practicing pathologist to diagnose this elusive, boutique entity. As we continue to gather data, the current classification scheme will either be solidified or discarded, with continuing attention paid to molecular changes and subsets of lesions. Clinicians treating patients with PDTC will need to become familiar with the diagnostic criteria for this diagnosis, constructing appropriate treatment modalities and follow up for these patients. With additional experience, the diagnostic model will be tested and ultimately found to be clinically relevant; and if not, it will be discarded for something deemed to be more rational.

\section{REFERENCES}

Akslen, L. A., and LiVolsi, V. A. (2000). Prognostic significance of histologic grading compared with subclassification of papillary thyroid carcinoma. Cancer 88, 1902-1908.

Asioli, S., Erickson, L. A., Righi, A., Jin, L., Volante, M., Jenkins, S., Papotti, M., Bussolati, G., and Lloyd, R. V. (2010). Poorly differentiated carcinoma of the thyroid: validation of the Turin proposal and analysis of IMP3 expression. Mod. Pathol. 23, 1269-1278.

Bongiovanni, M., Sadow, P. M., and Faquin, W.C. (2009). Poorly differentiated thyroid carcinoma: a cytologichistologic review. Adv. Anat. Pathol. 16, 283-289.

Carcangiu, M. L., Zampi, G., and Rosai, J. (1984). Poorly differentiated ("insular") thyroid carcinoma. Am. J. Surg. Pathol. 8, 655-668.

Chakravarty, D., Santos, E., Ryder, M., Knauf, J. A., Liao, X. H., West, B. L., Bollag, G., Kolesnick, R., Thin, T H., Rosen, N., Zanzonico, P., Larson, S. M., Refetoff, S. Ghossein, R., and Fagin, J.A. (2011). Small-molecule MAPK inhibitors restore radioiodine incorporation in mouse thyroid cancers with conditional BRAF activation. J. Clin. Invest. 121, 4700-4711.

Dettmer, M., Schmitt, A., Steinert, A., Steinert, H., Moch, H., Komminoth, P., and Perren, A. (2012). Poorly differentiated oncocytic thyroid carcinoma - diagnostic implications and outcome. Histopathology 60, 1045-1051.

Dettmer, M., Schmitt, A., Steinert, H., Haldemann, A., Meili, A., Moch, H., Komminoth, P., and Perren, A. (2011). Poorly differentiated thyroid carcinomas: how much poorly differentiated is needed? Am. J. Surg. Pathol. 35, 1866-1872.

Dobashi, Y., Sugimura, H., Sakamoto, A., Mernyei, M., Mori, M., Oyama, T., and Machinami, R. (1994). Stepwise participation of $\mathrm{p} 53$ gene mutation during dedifferentiation of human thyroid carcinomas. Diagn. Mol. Pathol. 3, 9-14.

Donghi, R., Longoni, A., Pilotti, S., Michieli, P., Della Porta, G., and Pierotti, M.A. (1993). Gene p53 mutations are restricted to poorly differentiated and undifferentiated carcinomas of the thyroid gland. J. Clin. Invest. 91, 1753-1760.

Fagin, J. A., Matsuo, K., Karmakar, A., Chen, D. L., Tang, S. H., and Koeffler, H. P. (1993). High prevalence of mutations of the p53 gene in poorly differentiated human thyroid carcinomas. J. Clin. Invest. 91, 179-184.

Garcia-Rostan, G., and Sobrinho-Simões, M. (2010). Poorly differentiated thyroid carcinoma: an evolving entity. Diagn. Histopathol. 17, 114-123.

Hiltzik, D., Carlson, D. L., Tuttle, R. M., Chuai, S., Ishill, N., Shaha, A., Shah, J. P., Singh, B., and Ghossein, R. A. (2006). Poorly differentiated thyroid carcinomas defined on the basis of mitosis and necrosis: a clinicopathologic study of 58 patients. Cancer 106, 1286-1295.

Kakudo, K., Bai, Y., Liu, Z., Li, Y., Ito, Y., and Ozaki, T. (2012). Classification of thyroid follicular cell tumors: with special reference to borderline lesions. Endocr. J. 59, 1-12.

Lavra, L., Ulivieri, A., Rinaldo, C., Dominici, R., Volante, M., Luciani, E., Bartolazzi, A., Frasca, F., Soddu, S. and Sciacchitano, S. (2009). Gal-3 is stimulated by gain-of-function p53 mutations and modulates chemoresistance in anaplastic thyroid carcinomas. J. Pathol. 218, 66-75.

Malaguarnera, R., Vella, V., Vigneri, R., and Frasca, F. (2007).p53 family proteins in thyroid cancer. Endocr. Relat. Cancer 14, 43-60. 
Nambiar, A., Pv, S., Susheelan, V., and Kuriakose, M. A. (2011). The concepts in poorly differentiated carcinoma of the thyroid: a review article. J. Surg. Oncol. $103,818-821$.

Nikiforov, Y. E. (2004). Genetic alterations involved in the transition from well-differentiated to poorly differentiated and anaplastic thyroid carcinomas. Endocr. Pathol. 15, 319-327.

Nikiforov, Y.E., and Nikiforova, M. N. (2011). Molecular genetics and diagnosis of thyroid cancer. Nat. Rev. Endocrinol. 7, 569-580.

Nucera, C., Porrello, A., Antonello, Z.A., Mekel, M., Nehs, M. A., Giordano, T. J., Gerald, D., Benjamin, L. E., Priolo, C., Puxeddu, E., Finn, S., Jarzab, B., Hodin, R. A., Pontecorvi, A., Nose, V., Lawler, J., and Parangi, S. (2010). B-Raf(V600E) and thrombospondin-1 promote thyroid cancer progression. Proc. Natl. Acad. Sci. U.S.A. 107, 10649-10654.

Quiros, R. M., Ding, H. G., Gattuso, P., Prinz, R. A., and $\mathrm{Xu}, \mathrm{X}$. (2005). Evidence that one subset of anaplastic thyroid carcinomas are derived from papillary carcinomas due to BRAF and p53 mutations. Cancer 103 , 2261-2268.

Ricarte-Filho, J. C., Ryder, M., Chitale, D. A., Rivera, M., Heguy, A., Ladanyi, M., Janakiraman, M., Solit, D., Knauf, J. A., Tuttle, R. M., Ghossein, R. A., and Fagin, J. A. (2009). Mutational profile of advanced primary and metastatic radioactive iodine-refractory thyroid cancers reveals distinct pathogenetic roles for BRAF, PIK3CA, and AKT1. Cancer Res. 69, 4885-4893.

Rivera, M., Ricarte-Filho, J., Patel, S., Tuttle, M., Shaha, A., Shah, J. P., Fagin, J.A., and Ghossein, R. A. (2010a). Encapsulated thyroid tumors of follicular cell origin with high grade features (high mitotic rate/tumor necrosis): a clinicopathologic and molecular study. Hum. Pathol. 41, 172-180.

Rivera, M., Ricarte-Filho, J., Tuttle, R. M., Ganly, I., Shaha, A., Knauf, J., Fagin, J., and Ghossein, R. (2010b). Molecular, morphologic, and outcome analysis of thyroid carcinomas according to degree of extrathyroid extension. Thyroid 20, 1085-1093.

Sakamoto, A., Kasai, N., and Sugano, H. (1983). Poorly differentiated carcinoma of the thyroid. A clinicopathologic entity for a high-risk group of papillary and follicular carcinomas of the thyroid gland. Cancer 52, 1849-1855.

Siegel, R., and Jemal, A. (2012). Cancer Facts and Figures 2012. Atlanta, GA: American Cancer Society, 10.

Siironen, P., Hagström, J., Mäenpää, H. O., Louhimo, J., Heikkilä, A., Heiskanen, I., Arola, J., and Haglund, C. (2010). Anaplastic and poorly differentiated thyroid carcinoma: therapeutic strategies and treatment outcome of 52 consecutive patients. Oncology 79, 400-408.

Sobrinho-Simões, M., Albores-Saavedra, J., Tallini, G., Santoro, M., Volante, M., Pilotti, S., Carcangiu, M. L., Papotti, M., Matias-Guiu, X., Guiter, G. E., Zakowski, M., and Sakamoto, A. (2004). "Poorly differentiated carcinoma," in World Health Organization Classification of Tumors: Pathology and Genetics of Tumours of Endocrine Organs, eds R. A. DeLellis, R. V. Lloyd, P. U. Heitz, and C. Eng (Lyon: IARC Press), 73-76.

Sobrinho-Simões, M., Sambade, C., Fonseca, E., and Soares, P. (2002). Poorly differentiated carcinomas of the thyroid gland: a review of the clinicopathologic features of a series of 28 cases of a heterogeneous, clinically aggressive group of thyroid tumors. Int. J. Surg. Pathol. 10, 123-131.
Tallini, G. (2011). Poorly differentiated thyroid carcinoma. Are we there yet? Endocr. Pathol. 22, 190-194.

Thompson, L. D. (2011). Hyalinizing trabecular adenoma of the thyroid gland. Ear Nose Throat J. 90, 416-417.

Volante, M., Collini, P., Nikiforov, Y. E., Sakamoto, A. Kakudo, K., Katoh, R., Lloyd, R. V., LiVolsi, V. A., Papotti, M., Sobrinho-Simoes, M., Bussolati, G., and Rosai, J. (2007). Poorly differentiated thyroid carcinoma: the Turin proposal for the use of uniform diagnostic criteria and an algorithmic diagnostic approach. Am. J. Surg. Pathol. 31, 1256-1264.

Volante, M., and Papotti, M. (2010). Poorly differentiated thyroid carcinoma: 5 years after the $2004 \mathrm{WHO}$ classification of endocrine tumours. Endocr. Pathol. 21, 1-6.

Volante, M., Rapa, I., Gandhi, M., Bussolati, G., Giachino, D., Papotti, M., and Nikiforov, Y.E. (2009). RAS mutations are the predominant molecular alteration in poorly differentiated thyroid carcinomas and bear prognostic impact. J. Clin. Endocrinol. Metab. 94, 4735-4741.

Received: 21 May 2012; accepted: 31 May 2012; published online: 21 June 2012.

Citation: Sadow PM and Faquin WC (2012) Poorly differentiated thyroid carcinoma: an incubating entity. Front. Endocrin. 3:77. doi: 10.3389/fendo.2012.00077

This article was submitted to Frontiers in Cancer Endocrinology, a specialty of Frontiers in Endocrinology Copyright $(2012$ Sadow and Faquin. This is an open-access article distributed under the terms of the Creative Commons Attribution Non Commercial License, which permits noncommercial use, distribution, and reproduction in other forums, provided the original authors and source are credited. 